\title{
A New Scalar Potential Formulation of the Magnetostatic Field Problem*
}

\author{
By Joseph E. Pasciak
}

\begin{abstract}
A new method for approximating magnetostatic field problems is given in this paper. The new method approximates the scalar potential for the magnetic intensity and is based on a volume integral formulation. The derivation of the new computational method uses the spectral properties of the relevant integral operator. The corresponding algorithm is similar to that obtained from coupled differential and boundary integral approaches. Convergence and stability theorems are proven. Finally, convergence results in actual computations are compared with results for the usual volume integral method used in GFUN3D.
\end{abstract}

1. Introduction. In this paper we define a new numerical method for magnetostatic field computation. We specifically consider the problem of calculating the nonlinear static field effects produced by the presence of iron-type materials. Applications for these problems occur in, for example, the design of accelerator and beam control magnets, fusion devices, electric motor design, and magnetic tape head design.

A variety of numerical methods for the solution of magnetostatics problems have been proposed and some general purpose codes have been produced [1], [2], [4], [5], [8], [12], [13]. For examples of magnetic field calculations, see [4], and the references therein. The magnetostatic field problem can be stated as an elliptic interface problem and the analysis presented in [3], [8] is applicable. Integral formulations of this problem have also been considered [6], [11].

In this paper, a new scalar potential integral formulation of the nonlinear magnetostatic field problem is defined and analyzed. To motivate the new method, we give an analysis for constant permeability problems which shows that the large errors in the iron domain observed with the usual volume integral method lie in subspaces perpendicular to gradient functions. The new method is designed to eliminate errors of this type while generalizing to a method for nonlinear problems which exhibits uniform convergence in the iron domain even as the permeability becomes uniformly large.

The new method is derived as a volume integral formulation which approximates the magnetic intensity $H$ in terms of the gradient of a scalar potential. It is then

Received December 16, 1982; revised August 1, 1983.

1980 Mathematics Subject Classification. Primary 35J60; Secondary 45E10.

*The submitted manuscript has been authored under contract DE-AC02-76CH00016 with the U. S. Department of Energy. Accordingly, the U. S. Government retains a nonexclusive, royalty-free license to publish or reproduce the published form of this contribution, or allow others to do so, for U. S. Government purposes. 
observed that the method can be implemented with computational work similar to a scalar potential-boundary integral formulation. In fact, if the iron domain is smooth enough so that certain results from classical potential theory hold then the method can be reformulated as a scalar potential-boundary integral method.

The new method seems particularly well suited for implementation as a general three dimension algorithm. Like the usual integral method, the mesh need only be described on the iron domain and hence the user input problem is simpler than that for differential methods which require mesh generation on the entire space. Like coupled differential and boundary integral approaches, only the evaluation of sparse matrix systems in the interior and full matrix systems on the boundary nodes are required. For implementation with piecewise linear functions on tetrahedra, the method uses the same singular integrals already evaluated in GFUN3D [1] and some easily evaluated gram or mass matrices associated with standard finite element problems. Off iron calculations are also economical since unlike GFUN3D, only the boundary nodes enter into the calculation.

The outline of the remainder of the paper is as follows. In Section 2, the magnetostatic field problem is defined and it is shown that the integral method exhibits high permeability errors (at least in constant permeability calculations). In Section 3, the new method is described and its efficient implementation is discussed. A nonlinear convergence and stability analysis is presented in Section 4. The analysis used is similar to that given by Friedman. In Section 5 results of actual computations are compared for the new approach and the usual volume integral method.

2. High Permeability Errors in Volume Integral Approaches. The basic equations for magnetostatics can be derived from Maxwell's equations and are given in terms of the magnetic inductance $B$, the magnetic intensity $H$, and the current density $J$,

$$
\operatorname{div} B=0, \quad \operatorname{curl} H=J .
$$

These equations are connected by a constitutive relation $B=\mu(x, H) H$ where the permeability $\mu$ is an experimentally determined nonlinear function of $H$ and position. In addition, the normal component of the $B$ field and the tangential components of the $H$ field are continuous across the boundary of regions with different permeability.

Typical magnetostatic field problems involve regions, which we shall denote $\Omega_{I}$, containing ferromagnetic material. We shall refer to the complement of the region $\Omega_{I}$ as the exterior even though it may have components which are surrounded by $\Omega_{I}$. Throughout the exterior region we always have $\mu(x, H)=1$.

One way of solving the above problem is by the introduction of the volume integral operator $G$ defined by

$$
G(M) \equiv \frac{-\nabla}{4 \pi} \int_{\Omega_{I}} M \cdot \nabla\left(\frac{1}{\left|x-x^{\prime}\right|}\right) d x^{\prime} .
$$

The integral operator $G$ maps vector functions defined on $\Omega_{I}$ into vector functions defined on $R^{3}$. Define the field due to sources $H_{S}$ as the field observed without the iron material present. $H_{S}$ is given by the integral relation

$$
H_{S}=\frac{1}{4 \pi} \int J \times \nabla \frac{1}{\left|x-x^{\prime}\right|} d x^{\prime} .
$$


We shall assume $H_{S}$ is known since (2.2) can be calculated analytically or approximated numerically to within reasonable precision.

Define the magnetization $M$ and the field due to magnetization $H_{M}$ by

$$
M=B-H \text { and } \quad H_{M}=H-H_{S} .
$$

It then can be shown that

$$
H_{M}=G(M) \text {. }
$$

By combining the above identities one can easily derive the nonlinear integral equation

$$
\bar{\chi}(x, H)^{-1} M(x)-G(M)(x)=H_{S}(x),
$$

where $\bar{\chi}(x, H)=\mu(x, H)-1$ and $H \equiv H(M)=H_{S}+G(M)$.

Before proceeding further, it is convenient to define some notation. Let the $L^{2}$ vector inner product on the iron domain be denoted by $(\cdot, \cdot)$, i.e.

$$
(u, v)=\int_{\Omega_{I}} u(x) \cdot v(x) d x
$$

The corresponding norm is denoted by $\|\cdot\|$.

There are various techniques available for discretizing (2.5) which lead to similar numerical algorithms. As a typical example consider the Galerkin discretization. First assume that we have defined approximation subspaces $\left\{\bar{S}_{h}\right\}$ in $L^{2}\left(\Omega_{I}\right)^{3}$. Typical subspaces can be defined, for example, by breaking the iron domain into subregions and considering subspaces of functions which are piecewise polynomial on each subregion in each component. The approximate solution $M_{h}$ is defined to be the unique function in $\bar{S}_{h}$ satisfying

$$
\left(\bar{\chi}^{-1} M_{h}-G M_{h}, \psi_{h}\right)=\left(H_{S}, \psi_{h}\right) \text { for all } \psi_{h} \text { in } \bar{S}_{h} .
$$

Here and in what follows we shall leave out the $(x, H)$ in $\bar{\chi}(x, H)$ for notational convenience.

The reason that (2.6) exhibits errors in high permeability calculations can be illustrated by considering constant permeability problems. We shall need some auxiliary subspaces of $L^{2}\left(\Omega_{I}\right)^{3}$. Define

$$
\begin{aligned}
& K=\left\{\psi \mid \psi=\nabla \phi \text { for some } \phi \in H^{1}\left(\Omega_{I}\right)\right\}, \\
& K_{0}=\left\{\psi \mid \psi=\nabla \phi \text { for some } \phi \in H_{0}^{1}\left(\Omega_{I}\right)\right\}, \\
& K_{H}=\text { subspace in } K \text { perpendicular to } K_{0}, \\
& N=\text { subspace in } L^{2}\left(\Omega_{I}\right)^{3} \text { perpendicular to } K .
\end{aligned}
$$

The space $H^{1}\left(\Omega_{I}\right)$ is the Sobolev space of order one on $\Omega_{I} . H_{0}^{1}\left(\Omega_{I}\right)$ is the subspace of $H^{1}\left(\Omega_{I}\right)$ whose boundary trace also vanishes (see [9], [10]).

The following results for the operator $G$ are proven in [7].

(i) $G$ is a nonpositive symmetric operator on $L^{2}\left(\Omega_{I}\right)^{3}$.

(ii) The spaces $K_{0}, K_{H}, N$ are invariant under $G$.

(iii) $N$ is the null space of $G$.

(iv) $G$ is minus the identity on $K_{0}$.

(v) The eigenvalues of $G$ on $K_{H}$ lie in the interval $[-1,-\varepsilon]$ where $0<\varepsilon \leqslant 1$ is a positive constant which depends only upon the domain $\Omega_{I}$. 
We consider the constant permeability problem

$$
\frac{1}{\mu_{0}-1} M-G(M)=H_{S}
$$

For illustration, we shall assume that the integral operator $G$ can be calculated exactly, however small errors due to numerical quadrature or discretization are made in the approximation $\tilde{H}_{S}$ of $H_{S}$. Thus we consider the approximate problem

$$
\frac{1}{\mu_{0}-1} M-G(M)=\tilde{H}_{S} \text {. }
$$

The errors in $\tilde{H}_{S}$ will in general produce small error components which lie in the space $N$. Error components in $N$ are then amplified by $\left(\mu_{0}-1\right)$ in the solution $M$. Observe that from the spectral properties of $G$, the components of $H_{S}$ in $K$ are only increased by a factor of at most $\varepsilon^{-1}$. Thus as $\mu_{0}$ gets large, the null space errors will eventually dominate the true solution.

From the above discussion, it is clear that high permeability errors can be reduced in constant permeability problems by projecting the approximate field due to sources or the resultant magnetization into $K$. For nonconstant permeability, it is not sufficient to project $\tilde{H}_{S}$ or the resultant magnetization onto $K$. The method described in the next section has convergence estimates which are independent of the permeability as the permeability gets uniformly large and thus succeeds in reducing these errors while generalizing to variable and nonlinear $\mu$ problems.

3. The New Method. The new method is described in this section. Using the properties of the operator $G$, an implementation of this method is given which only requires sparse matrix operations in the interior of the iron domain and full matrix calculation from boundary to boundary elements. The resulting discretization is similar to a coupled scalar potential-boundary integral type formulation. We believe that this approach is computationally competitive with any existing approach.

The arguments given at the end of Section 2 imply that with constant permeability, a necessary and sufficient condition for the $B$ field to be uniformly bounded as the permeability becomes large is that the field due to sources $H_{S}$ be in $K$. Thus we shall assume throughout this paper that $H_{S}$ is in $K$. Note that this condition is satisfied when the average current enclosed by every loop in the iron domain is zero. From Eqs. (2.3) and (2.4) we see that $H$ is also in $K$. The new method is then defined to be the $H$ formulation of the usual volume integral method posed on subspaces of gradient functions. Thus we seek the solution of the problem

$$
(I-G \bar{\chi}) H=H_{S} \quad \text { where } H \text { is in } K .
$$

The reason that (3.1) is an improvement to (2.5) can now be stated. As long as the ratio $\bar{\chi}_{\max } / \bar{\chi}_{\min }$ is not large then the condition number of the operator $(I-G \bar{\chi})$ is bounded independent of the magnitude of $\bar{\chi}$. Thus one expects that the corresponding approximation method converges with rates that are also independent of the magnitude of $\bar{\chi}$.

Let $P$ denote the $L^{2}\left(\Omega_{I}\right)^{3}$ orthogonal projection onto $K$. Since $K$ is the orthogonal complement of the null space $N$, the above problem is equivalent to the problem

$$
(I-G P \bar{\chi}) H=H_{S} \text { for } H \text { in } K .
$$

To discretize the above problem, we first introduce approximation subspaces $\left\{K_{h}\right\}$ of $K$. The natural way to define $K_{h}$ is by first defining a standard scalar-valued 
approximation subspace $\left\{S_{h}\right\}$ on $\Omega_{I}$ and then defining

$$
K_{h}=\left\{\nabla \phi \mid \phi \text { in } S_{h}\right\} .
$$

An example of $S_{h}$ is given by triangulating the domain $\Omega_{I}$ and letting $S_{h}$ be the space of functions which are continuous on $\Omega_{I}$ and piecewise linear on the triangles. For such subspaces, the functions in $K_{h}$ are piecewise constant on the triangles and the number of basis elements is the number of triangular vertices (nodes) minus one.

The new discrete method is then essentially the Galerkin method applied to (3.2). Specifically, the approximate solution $H_{h}$ is defined as the solution of the problem

$$
\left(H_{h}-G P_{h} \bar{\chi}\left(x, H_{h}\right) H_{h}, \psi_{h}\right)=\left(H_{S}, \psi_{h}\right) \text { for } \psi_{h} \text { in } K_{h} \text {. }
$$

The operator $P_{h}$ is defined to be the $L^{2}\left(\Omega_{I}\right)^{3}$ orthogonal projection onto $K_{h}$. We shall see in the next section that problem (3.3) has a unique solution under physically reasonable assumptions on the permeability function. In addition, error and stability estimates for the solution in the iron domain will be given which are independent of the permeability as the permeability gets large (Theorem 2).

We next consider implementation of (3.3). First observe that (3.3) can be written

$$
H_{h}-G_{h} P_{h}\left(\bar{\chi} H_{h}\right)=P_{h} H_{S},
$$

where the discrete operator $G_{h}$ is defined by $G_{h} \psi_{h}=\theta_{h}$ and $\theta_{h}$ satisfies

$$
\left(\theta_{h}, \omega_{h}\right)=\left(G \psi_{h}, \omega_{h}\right) \text { for all } \omega_{h} \text { in } K_{h} \text {. }
$$

We shall solve problem (3.4) by matrix iterative techniques. Note that to solve (3.4) with iterative techniques it is only necessary to evaluate the action of the operator on the left-hand side on functions in $K_{h}{ }^{* *}$ Thus we must be able to evaluate $P_{h}$ and $G_{h}$. To evaluate $P_{h}$ it is only necessary to solve the standard sparse matrix problem associated with the Neumann problem on the subspace $S_{h}$ or equivalently solve the $L^{2}\left(\Omega_{I}\right)^{3}$ Gram matrix for the subspace $K_{h}$. The computation of $G_{h}$ leads to the same matrix problem as for $P_{h}$ and the evaluation of the quantities

$$
\left(G_{\psi h}, \xi_{i}\right)=F_{i}
$$

as $\xi_{i}$ ranges over all of the finite element basis functions of $K_{h}$. A priori, it looks like the evaluation of the quantities in (3.6) requires the multiplication of a full matrix times a vector with dimension equal to the number of iron nodes. We shall demonstrate that (3.6) can be evaluated in far fewer operations. The key observation is that from the spectral results for the operator $G$ we have

$$
(G+I) \psi=0 \text { for } \psi \text { in } K_{0} .
$$

We shall assume that we have a finite element basis for the functions in $S_{h}$ for which functions can be represented

$$
\psi_{h}=\sum_{i=1}^{N_{0}} c_{i} \tau_{i}+\sum_{i=1}^{N_{b}} d_{i} \beta_{i} .
$$

Here the functions $\left\{\tau_{i}\right\}$ are zero on $\partial \Omega$ and the functions $\left\{\beta_{i}\right\}$ are nonzero only near the boundary. We also define $S_{h}^{0}$ and $S_{h}^{b}$ by

$$
\begin{aligned}
& S_{h}^{0}=\operatorname{span}\left\{\tau_{i}\right\}, \quad i=1, \ldots, N_{0} \\
& S_{h}^{b}=\operatorname{span}\left\{\beta_{i}\right\}, \quad i=1, \ldots, N_{b} .
\end{aligned}
$$

\footnotetext{
**For a description of how this can be done with the similar problem (2.5) see [11].
} 
Note that the dimension of $S_{h}^{0}$ is essentially the number of interior nodes while the dimension of $S_{h}^{b}$ is essentially the number of boundary nodes. Let $\psi_{h}$ and $\theta_{h}$ be functions in $K_{h}$; then $\psi_{h}=\nabla \phi_{h}$ and $\theta_{h}=\nabla \eta_{h}$ for some functions $\phi_{h}$ and $\eta_{h}$ in $S_{h}$. Decompose $\phi_{h}$ and $\eta_{h}$ by

$$
\phi_{h}=\phi_{h}^{0}+\phi_{h}^{b} \quad \text { and } \quad \eta_{h}=\eta_{h}^{0}+\eta_{h}^{b},
$$

where $\phi_{h}^{0}, \eta_{h}^{0}$ (resp. $\phi_{h}^{b}, \eta_{h}^{b}$ ) are in $S_{h}^{0}$ (resp. $S_{h}^{b}$ ). Then (3.7) implies the identity

$$
\left(G_{h} \psi_{h}, \theta_{h}\right)=\left((G+I) \nabla \phi_{h}^{b}, \nabla \eta_{h}^{b}\right)-\left(\psi_{h}, \theta_{h}\right) .
$$

Thus for any given function $\psi_{h}$, the evaluation of (3.6) requires a sparse matrix evaluation to compute

$$
\left(\psi_{h}, \xi_{i}\right) \text { for } i=1, \ldots, N \text {, }
$$

and a full $N_{b} \times N_{b}$ matrix evaluation to compute

$$
\left((G+I) \nabla \phi_{h}^{b}, \nabla \beta_{i}\right) \text { for } i=1, \ldots, N_{b} .
$$

We finally note that field approximation outside the iron is calculated from the formula

$$
B_{h}(x)=\left(H_{S}+G M_{h}\right)(x) \text { where } M_{h}=P_{h} \bar{\chi} H_{h} .
$$

Decomposing $M_{h}=M_{h}^{0}+M_{h}^{b}$ and noting that $G$ of functions in $K_{0}$ is zero outside of $\Omega_{I}$ gives that

$$
B_{h}(x)=\left(H_{S}+G M_{h}^{b}\right)(x) \text { for } x \text { not in } \Omega_{I} \text {. }
$$

Thus only the boundary nodes need be used to calculate fields outside of the iron domain.

4. Analysis of the Discrete Method. The new method is analyzed in this section. Convergence and stability results are given under certain monotonicity assumptions for the susceptibility function $\bar{\chi}$. We demonstrate that these monotonicity assumptions hold for the susceptibility functions of isotropic iron materials satisfying physically reasonable assumptions.

For our existence theorems we shall require that $\bar{\chi}(x, H) H$ be strongly monotone and Lipschitz continuous in $L^{2}\left(\Omega_{I}\right)^{3}$, that is

(a.1) $\left(\bar{\chi}\left(x, H_{1}\right) H_{1}-\bar{\chi}\left(x, H_{2}\right) H_{2}, H_{1}-H_{2}\right) \geqslant C_{0}\left(H_{1}-H_{2}, H_{1}-H_{2}\right)$

and

$$
\left\|\bar{\chi}\left(x, H_{1}\right) H_{1}-\bar{\chi}\left(x, H_{2}\right) H_{2}\right\| \leqslant C_{1}\left\|H_{1}-H_{2}\right\|
$$

for constants $C_{0} \geqslant 0$ and $C_{1}>0$. Note that $\bar{\chi}(x, H) H$ is the magnetization corresponding to the field $H$. For many applications, $H$ is a priori bounded and $C_{0}$ may be comparable to $C_{1}$.

The following two theorems give stability and convergence results for the new method and its discretization.

THEOREM 1. If Assumptions (a.1) and (a.2) hold then (3.1) has a unique solution.

THEOREM 2. If Assumptions (a.1) and (a.2) hold then (3.3) has a unique solution $H_{h}$. Furthermore, if $H$ solves (3.1) and $\delta=\inf _{\psi \in K_{h}}\|H-\psi\|$ then

$$
\left\|H-H_{h}\right\| \leqslant \delta\left\{1+\frac{\varepsilon^{-1 / 2}+C_{1}}{1+C_{0} \varepsilon}\right\} .
$$


From (4.1) we see that the discrete method gives quasi optimal convergence to $H$ in $L^{2}\left(\Omega_{I}\right)^{3}$. As we shall demonstrate, for many applications the ratio $C_{1} / C_{0}$ is bounded even though the permeability is large and thus the constant

$$
C(\bar{\chi}) \equiv 1+\frac{\varepsilon^{-1 / 2}+C_{1}}{1+C_{0} \varepsilon}
$$

is also bounded.

We shall prove only Theorem 2 . The proof of Theorem 1 is similar to the existence and uniqueness part of Theorem 2. The techniques used in these proofs are similar to those given by Friedman in [5].

Proof of Theorem 2. We shall first set up some notation for the proof. Introduce the additional inner products on $K$ and $K_{h}$ by

$$
\begin{array}{ll}
\langle\langle u, v\rangle\rangle \equiv-\left(G^{-1} u, v\right) & \text { for } u, v \text { in } K, \\
\langle\langle u, v\rangle\rangle_{h} \equiv-\left(G_{h}^{-1} u, v\right) & \text { for } u, v \text { in } K_{h} .
\end{array}
$$

By (v) and (3.5) the corresponding norms $[\cdot]$ and $[\cdot]_{h}$ satisfy the estimates

$$
\|u\|^{2} \leqslant[u]^{2} \leqslant \varepsilon^{-1}\|u\|^{2}
$$

and

$$
\|u\|^{2} \leqslant[u]_{h}^{2} \leqslant \varepsilon^{-1}\|u\|^{2}
$$

Also, for $H^{i} \in K$ and $H_{h}^{i} \in K_{h}$ let

$$
M^{i} \equiv \bar{\chi}\left(x, H^{i}\right) H^{i} \quad \text { and } \quad M_{h}^{i} \equiv \bar{\chi}\left(x, H_{h}^{i}\right) H_{h}^{i}
$$

and finally

$$
R_{h} \theta \equiv \theta-G_{h} P_{h} \bar{\chi}(x, \theta) \theta \quad \text { and } \quad R \theta \equiv \theta-G \bar{\chi}(x, \theta) \theta .
$$

Our approximate problem (3.3) can then be written

$$
R_{h} H_{h}=P_{h} H_{S} \text {. }
$$

We obviously have for $H_{h}^{1}$ and $H_{h}^{2}$ in $K_{h}$

$$
\begin{aligned}
& \left\langle\left\langle R_{h} H_{h}^{1}-R_{h} H_{h}^{2}, H_{h}^{1}-H_{h}^{2}\right\rangle\right\rangle_{h} \\
& \quad=\left\langle\left\langle H_{h}^{1}-H_{h}^{2}, H_{h}^{1}-H_{h}^{2}\right\rangle\right\rangle_{h}+\left(M_{h}^{1}-M_{h}^{2}, H_{h}^{1}-H_{h}^{2}\right) .
\end{aligned}
$$

By (a.1)

$$
\left(M_{h}^{1}-M_{h}^{2}, H_{h}^{1}-H_{h}^{2}\right) \geqslant C_{0}\left\|H_{h}^{1}-H_{h}^{2}\right\|^{2} \geqslant C_{0} \varepsilon\left[H_{h}^{2}-H_{h}^{1}\right]_{h}^{2} .
$$

Combining this with (a.2) gives

$$
\begin{aligned}
\left(1+\varepsilon C_{0}\right)\left[H_{h}^{1}-H_{h}^{2}\right]_{h}^{2} & \leqslant\left\langle\left\langle R_{h} H_{h}^{1}-R_{h} H_{h}^{2}, H_{h}^{1}-H_{h}^{2}\right\rangle\right\rangle_{h} \\
& \leqslant\left(1+C_{1}\right)\left[H_{h}^{1}-H_{h}^{2}\right]_{h}^{2} .
\end{aligned}
$$

By the standard theory of monotone operators [14], (4.4) implies existence and uniqueness of the solution of problem (3.3). An estimate analogous to (4.4) can be proved for the operator $R$, the norm [ $\cdot$, and the inner product $\langle\langle\rangle$,$\rangle hence proving$ Theorem 1.

We next derive error estimates. Let $\tilde{H}=P(H)$; then from the definition of $\delta$ we obviously have

$$
\|\tilde{H}-H\|=\delta .
$$


By (4.4), (3.1) and (3.3)

$$
\begin{aligned}
& \left(1+\varepsilon C_{0}\right)\left[\tilde{H}-H_{h}\right]_{h}^{2} \leqslant\left\langle\left\langle R_{h} \tilde{H}-R_{h} H_{h}, \tilde{H}-H_{h}\right\rangle\right\rangle_{h} \\
& \quad \leqslant\left\langle\left\langle R_{h} \tilde{H}-P_{h} R H, \tilde{H}-H_{h}\right\rangle\right\rangle_{h} \\
& \quad \leqslant\left\langle\left\langle\tilde{H}-H, \tilde{H}-H_{h}\right\rangle\right\rangle_{h}-\left\langle\left\langle G_{h} \tilde{M}-P_{h} G M, \tilde{H}-H_{h}\right\rangle\right\rangle_{h} \equiv T_{1}-T_{2} .
\end{aligned}
$$

Here $\tilde{M} \equiv \bar{\chi}(x, \tilde{H}) \tilde{H}$.

By the Schwarz inequality and (4.5)

$$
\left|T_{1}\right| \leqslant \varepsilon^{-1 / 2} \delta\left[\tilde{H}-H_{h}\right]_{h} .
$$

From (3.5), $P_{h} G=G_{h}$ and thus (a.2) and (4.3) imply

$$
\left|T_{2}\right|=\left|\left(\bar{\chi}(x, \tilde{H}) \tilde{H}-\bar{\chi}(x, H) H, \tilde{H}-H_{h}\right)\right| \leqslant C_{1} \delta\left\|\tilde{H}-H_{h}\right\| \leqslant C_{1} \delta\left[\tilde{H}-H_{h}\right]_{h} .
$$

Combining the above inequalities gives

$$
\left[\tilde{H}-H_{h}\right]_{h} \leqslant \frac{\left(\varepsilon^{-1 / 2}+C_{1}\right)}{\left(1+\varepsilon C_{0}\right)} \delta .
$$

Using (4.3), the triangle inequality and (4.5) gives estimate (4.1) and completes the proof of the theorem.

We next consider isotropic materials where

$$
\bar{\chi}(x, H)=\chi(|H(x)|) .
$$

We assume that $\chi$ is a nonnegative scalar function satisfying

$$
\begin{gathered}
C_{0}(b-a)^{2} \leqslant\{\chi(a) a-\chi(b) b\}\{a-b\}, \\
|\chi(a) a-\chi(b) b| \leqslant C_{1}|a-b|
\end{gathered}
$$

for nonnegative $a$ and $b$. Note that (a.3) with $C_{0}=0$ is satisfied if $\chi$ is nondecreasing.

Proposition 1. Let $\bar{\chi}$ be defined by (4.8) with a susceptibility function $\chi$ satisfying (a.3)-(a.4). Then (a.1) and (a.2) hold.

Proof. Let $Q(x, y)$ be defined by

$$
Q(x, y) \equiv\{\chi(|x|) x-\chi(|y|) y\}\{x-y\} \text { for } x, y \text { in } R^{3} .
$$

From obvious properties of the integral, it is sufficient to show that

$$
Q(x, y) \geqslant C_{0}|x-y|^{2}
$$

to verify (a.1) and that

$$
|\chi(|x|) x-\chi(|y|) y| \leqslant C_{1}|x-y|
$$

to verify (a.2). We may assume without loss of generality that $|x| \geqslant|y|$ and that $|x|>0$. Let

$$
e=x /|x|
$$

then

$$
y=|y|(\lambda e+w),
$$

where $w$ is perpendicular to $e$ and $|\lambda| \leqslant 1$. Let $F_{x}=\chi(|x|)|x|$ and $F_{y}=\chi(|y|)|y|$. Using (4.11) and (4.12) it is straightforward to compute

$$
Q(x, y)=\left(F_{x}-F_{y}\right)(|x|-|y|)+(1-\lambda)\left(F_{x}|y|+F_{y}|x|\right) .
$$


Thus (4.9) follows immediately from (4.13) and (a.3).

Let $\omega \equiv|\chi(|x|) x-\chi(|y|) y|$. Using (4.11) and (4.12), we compute

$$
\omega^{2}=\lambda\left(F_{x}-F_{y}\right)^{2}+(1-\lambda)\left(F_{x}^{2}+F_{y}^{2}\right)
$$

and

$$
|x-y|^{2}=\lambda(|x|-|y|)^{2}+(1-\lambda)\left(|x|^{2}+|y|^{2}\right) .
$$

Thus (a.2) follows from (a.4) which completes the proof of the proposition.

5. Applications and Numerical Results. In this section, computational results are given which show that the new approach produces noticeable improvements over the usual integral method for high permeability calculation in the iron region. In addition, we consider a typical $B-H$ curve and see that the constants $C_{0}$ and $C_{1}$ of (a.1) and (a.2) are comparable in low field calculations. Thus for this application, the estimate of Theorem 2 is good even though the permeability is large.

Our computational examples use an annular iron region in two dimensions of inner radius one and outer radius two. We shall consider constant permeability calculations with source fields for which analytic results are easily obtained. We only consider the errors in the iron domain; consequently, errors obtained may seem unreasonably large. Results from these codes show substantial improvement off the iron domain and hence do not contradict off iron convergence results for GFUN quoted elsewhere.

For the usual integral calculation we employ the Galerkin type discretization corresponding to (2.6). The annular domain is partitioned into $N_{r} \times N_{\theta}$ quadrilateral elements and a system with $3 \cdot N_{r} \cdot N_{\theta}$ unknowns is solved. Unknown field quantities are approximated by piecewise constant vector functions and hence the convergence is asymptotically (as $N_{r}$ and $N_{\theta}$ tend to infinity) first order.

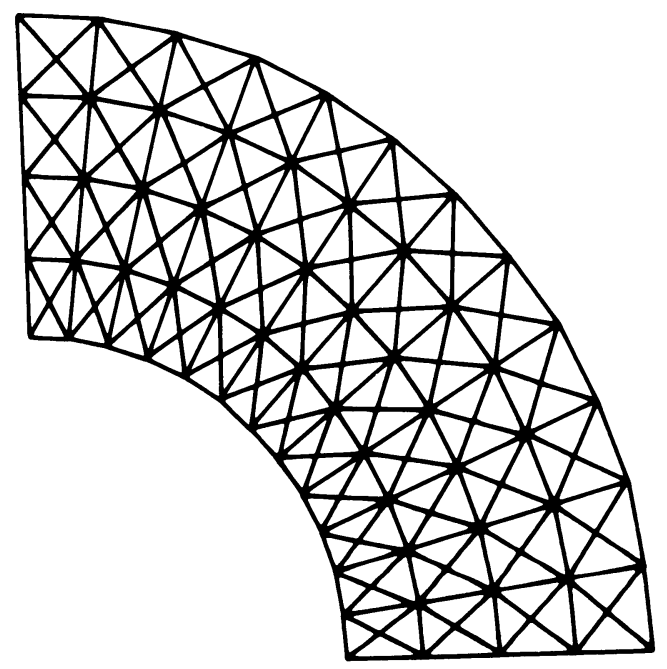

FIGURE 1

The first quadrant mesh used with the new method 


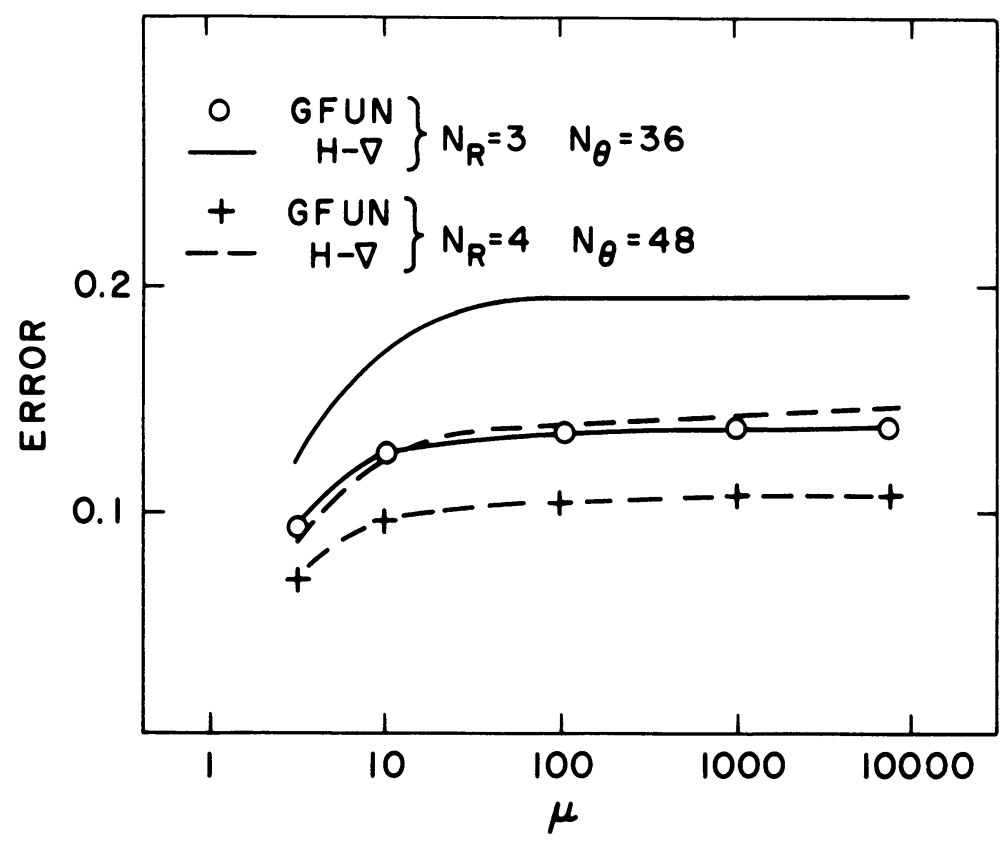

FIGURE 2

Normalized max norm error for $H-\nabla$ and GFUN (constant $H_{S}$ and no integration error)

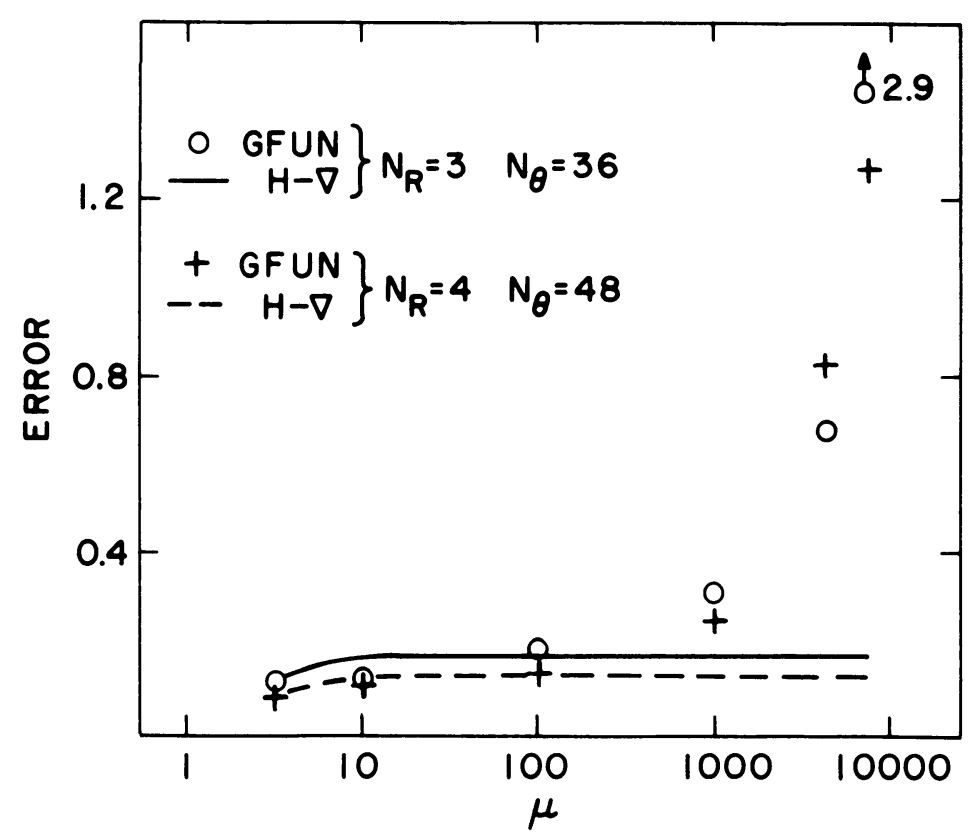

Figure 3

Normalized max norm error for $H-\nabla$ and GFUN (constant $H_{S}$ and random integration error) 


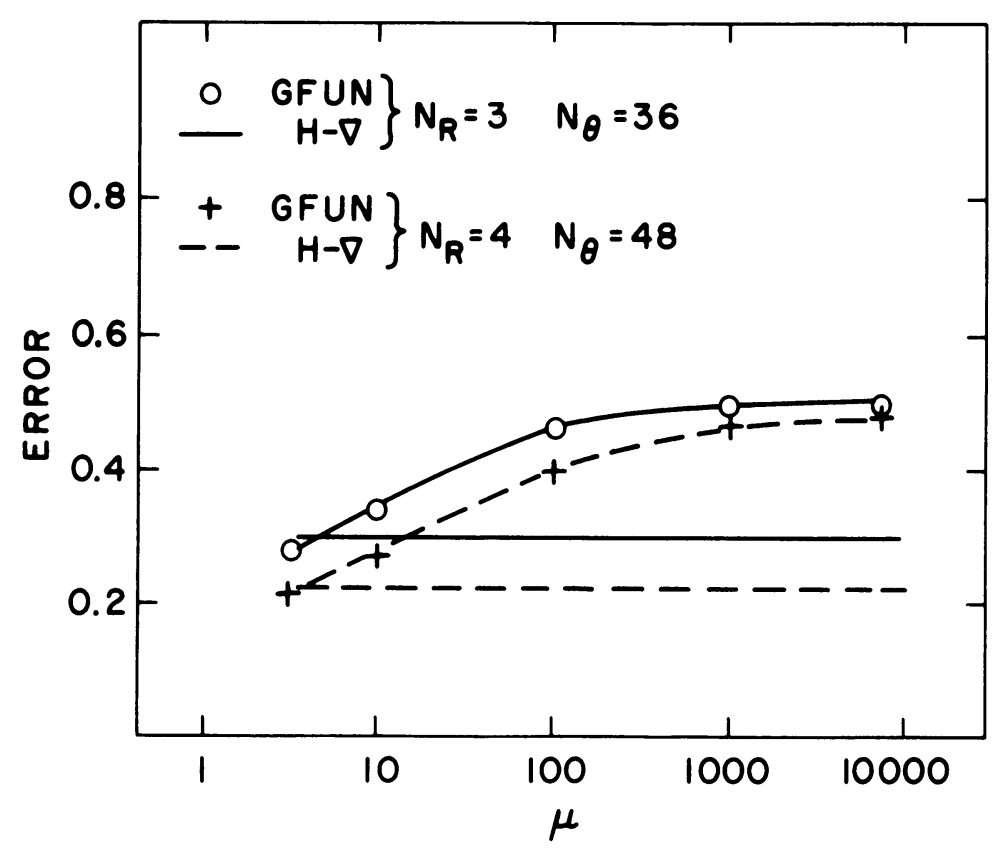

FIGURE 4

Normalized norm error for $H-\nabla$ and GFUN

( $H_{S}$ given by second harmonic)

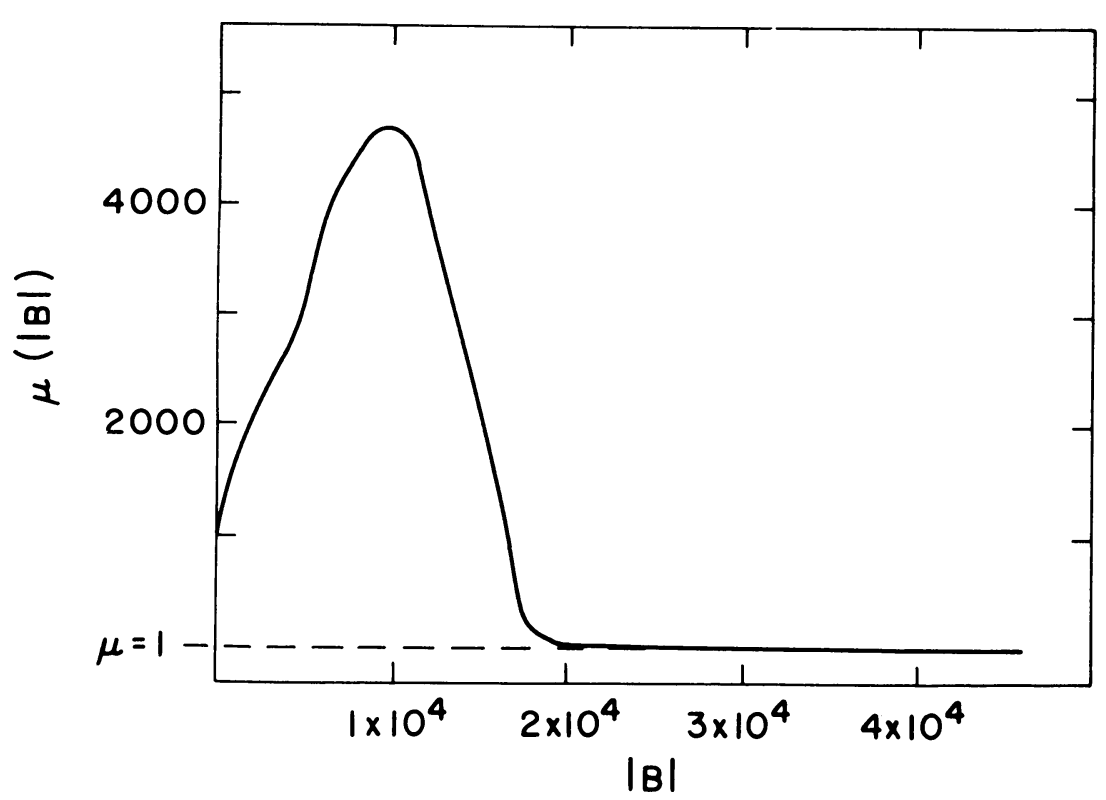

FIGURE 5

Permeability for $C B A$ iron 
For the new method which we shall sometimes denote $H-\nabla$, the annular domain is first partitioned into $N_{r} \times N_{\theta}$ quadrilateral regions and then each quadrilateral region is split into four triangular elements (see Figure 1). The subspace $S_{h}$ is defined to be functions which are piecewise linear on the triangles and continuous on the entire iron domain. Unknown field quantities are approximated by functions which are piecewise constant on the elements and, consequently, the convergence is asymptotically first order. The field approximation requires the solution of a system with $\left(2 N_{r}+1\right) \cdot N_{\theta}-1$ unknowns.

The first set of calculations are for an $H_{S}$ field which is constant and points in the $x$ direction. Figure 2 compares the maximum norm difference of the computed and the analytic $B$ field on the iron domain for the usual integral method and the new approach. This example is really rather special in that the numerical quadratures on the right-hand side of (2.6) and (3.3) produce exact integration results. Thus the argument in Section 2 is not valid and both the integral approach and the new approach give convergence results which are independent of the permeability.

The errors of the two methods are compared in Figure 3 for a constant $H_{S}$ calculation with a numerical integration error of magnitude $1 / N_{\theta}$ added in the computation of the quantities on the right-hand side of (2.6) and (3.3). Note that both methods are stable with respect to these small quadrature errors in low permeability calculation however a drastic difference in convergence is seen in high permeability calculations with the integral method where these small quadrature errors are blown way out of proportion. As theoretically predicted the new method remains stable and convergent throughout the entire permeability region.

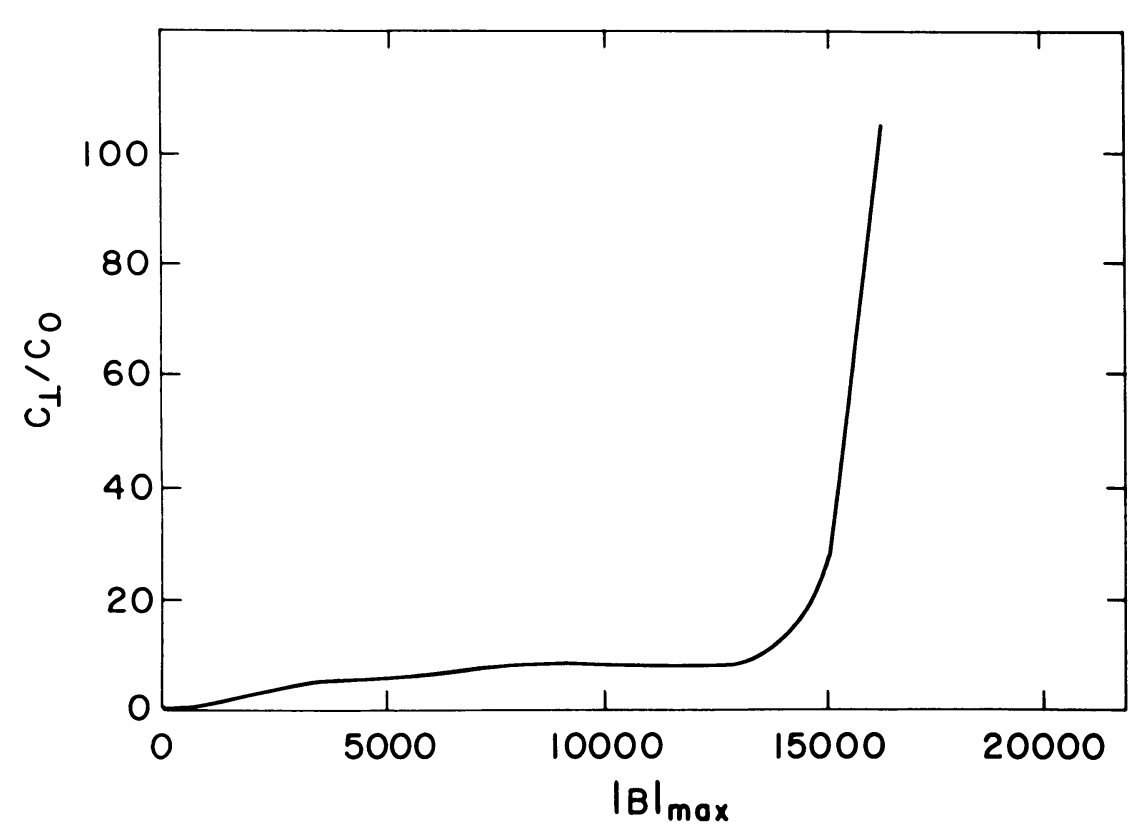

Figure 6

Bound for $C_{1} / C_{0}$ a function of $B_{\max }$ for the permeability of Figure 5 
For the third example (Figure 4), errors are considered for calculations with conductor fields defined by the second harmonic. That is,

$$
H_{S}=\left(x^{2}-y^{2},-2 x y\right) \text {. }
$$

These calculations are more representative of the situation encountered in actual applications since the quadrature rules give only an approximation to the data for (2.6) and (3.3). Note that the error exhibited by the new method is independent of the permeability. The errors observed with the usual integral method increase with permeability. Note also that a $75 \%$ reduction in the mesh size leads to the predicted $75 \%$ error reduction with the new approach while a $75 \%$ reduction in mesh with the integral method leads to only a 95\% error reduction with large $\mu$.

We next apply Proposition 1 to obtain bounds for the ratio $C 1 / C 0$ as a function of the maximum field value $|B|_{\max }$ for a typical $B-H$ curve. Figure 5 gives the measured $B-H$ curve for the iron used in the "Colliding Beam Accelerator" (CBA) superconducting magnets developed at Brookhaven National Laboratory. Figure 6 gives the corresponding bound for $C 1 / C 0$. Note that the ratio remains relatively small so long as $|B|_{\max }$ is less than about 15000 Gauss. Thus for low field applications, Theorem 2 guarantees uniform (independent of the permeability) convergence of the nonlinear magnetostatic field problem in the iron domain.

Applied Mathematics Department, 515

Brookhaven National Laboratory

Upton, New York 11973

1. A. G. Armstrong, A. M. Collie, C. J. Diserens, N. J. Newman, M. J. Simkin \& C. W. Trowbridge, New Developments in the Magnet Design Program GFUN, Rutherford Laboratory Report No. RL-79-097.

2. J. H. BRAmble \& J. E. PASCiaK, "A new computational approach for the linearized scalar potential formulation of the magnetostatic field problem," IEEE Trans. Mag. v. Mag-18. 1982, pp. 357-361.

3. F. Brezzi, C. Johnson \& J. C. Nedelec, On the Coupling of Boundary Integral and Finite Element Methods, Rapport interne \#39, École Polytechnique, 1978.

4. M. V. K. Chari \& P. P. Silvester (Editors), Finite Elements in Electrical and Magnetic Field Problems, Wiley, New York, 1980.

5. M. J. Friedman, "Finite element formulation of the general magnetostatic problem in the space of generalized solenoidal vector functions," Math. Comp., v. 43, 1984, pp. 415-431.

6. M. J. FrIEDMAN, "Mathematical study of the nonlinear singular integral magnetic field equation I," SIAM J. Appl. Math., v. 39, 1980, pp. 14-20.

7. M. J. Friedman \& J. E. PASCIAK, "Spectral properties for the magnetization integral operator," Math. Comp., v. 43, 1984, pp. 447-453.

8. R. Glowinski \& A. Marrocco, "Analyse numérique du champ magnétique d'un alternateur par éléments finis et surrelaxation ponctuelle non linéaire," Comput. Methods Appl. Mech. Engrg., v. 3, 1974 , pp. 55-85.

9. J. L. Lions \& E. Magenes, Non-Homogeneous Boundary Value Problems and Applications, Springer-Verlag, New York, 1972.

10. J. NeCaS, Les Méthodes Directes en Théorie des Équations Elliptiques, Masson, Paris, 1967.

11. J. PASCIAK, "An iterative algorithm for the volume integral method for magnetostatics problems," Comput. Math. Appl., v. 8, 1982, pp. 283-290.

12. J. Simkin \& C. W. Trowbridge, Three Dimensional Computer Program (TOSCA) for Nonlinear Electromagnetic Fields, Rutherford Laboratory Report No. RL-79-097.

13. C. W. Trowbridge, Progress in Magnet Design by Computers, Proc. Fourth Internat. Conf. Magnet. Tech., U.S. Atomic Energy Commission Rep. Conf-720908, 1972, pp. 555-565.

14. M. M. VAINBERG, Variational Method and Method of Monotone Operators in the Theory of Nonlinear Equations, Wiley, New York, 1973.

15. V. S. Vladimirov, Equations of Mathematical Physics, Marcel Dekker, New York, 1971. 